\title{
Displacement analysis of myocardial mechanical deformation (DIAMOND) reveals segmental susceptibility to doxorubicin-induced injury and regeneration
}

Junjie Chen, ${ }^{1}$ Yichen Ding, ${ }^{2}$ Michael Chen, ${ }^{1}$ Jonathan Gau, ${ }^{2}$ Nelson Jen, ${ }^{2}$ Chadi Nahal, ${ }^{1}$ Sally Tu, ${ }^{3}$ Cynthia Chen, ${ }^{1}$ Steve Zhou, ${ }^{2}$ Chih-Chiang Chang, ${ }^{1}$ Jintian Lyu, ${ }^{1}$ Xiaolei Xu, ${ }^{4}$ Tzung K. Hsiai, ${ }^{1,2}$ and René R. Sevag Packard²

'Department of Bioengineering, Henry Samueli School of Engineering and Applied Sciences, ${ }^{2}$ Division of Cardiology, Department of Medicine, David Geffen School of Medicine, ${ }^{3}$ Department of Neuroscience, College of Letters and Science, University of California, Los Angeles, California, USA. ${ }^{4}$ Department of Biochemistry and Molecular Biology, Mayo Clinic College of Medicine, Rochester, Minnesota, USA.

Zebrafish are increasingly utilized to model cardiomyopathies and regeneration. Current methods evaluating cardiac function have known limitations, fail to reliably detect focal mechanics, and are not readily feasible in zebrafish. We developed a semiautomated, open-source method displacement analysis of myocardial mechanical deformation (DIAMOND) - for quantitative assessment of 4D segmental cardiac function. We imaged transgenic embryonic zebrafish in vivo using a light-sheet fluorescence microscopy system with 4D cardiac motion synchronization. Our method permits the derivation of a transformation matrix to quantify the time-dependent $3 D$ displacement of segmental myocardial mass centroids. Through treatment with doxorubicin, and by chemically and genetically manipulating the myocardial injury-activated Notch signaling pathway, we used DIAMOND to demonstrate that basal ventricular segments adjacent to the atrioventricular canal display the highest $3 \mathrm{D}$ displacement and are also the most susceptible to doxorubicin-induced injury. Thus, DIAMOND provides biomechanical insights into in vivo segmental cardiac function scalable to high-throughput research applications.

Authorship note: $\mid \mathcal{C}$ and $Y D$ contributed equally to this work.

Conflict of interest: The authors have declared that no conflict of interest exists.

Copyright: () 2019 American Society for Clinical Investigation

Submitted: October 5, 2018 Accepted: February 27, 2019 Published: April 18, 2019

Reference information: /CI Insight. 2019;4(8):e125362. https://doi. org/10.1172/jci.insight.125362

\section{Introduction}

Chemotherapy-induced cardiac toxicity and ensuing heart failure are the main reason for chemotherapy discontinuation (1). Anthracyclines such as doxorubicin are among the most effective chemotherapeutic agents; however, they lead to frequent cardiovascular complications, including heart failure (2). While human induced pluripotent stem cells (hiPSCs) have been proposed to assess chemotherapy-induced cardiotoxicity (3), experimental models such as zebrafish (Danio rerio) heal by regenerating myocardium with highly conserved developmental genes (4), allowing for in vivo assessment of chemotherapy and cardiac function in an integrated organ system (5). In addition, zebrafish are increasingly utilized to model different types of heart failure and regeneration, for drug development, and to search for genetic modifiers (6-9). However, their small heart size hinders precise functional and structural assessments, necessitating advanced light-sheet fluorescence microscopy (LSFM) with high spatiotemporal resolution for in vivo imaging of cardiac development, injury, and regeneration (10-15).

Myocardial mechanical properties are critical determinants of cardiac function (16), with regional variations in myocardial contractility demonstrated within infarcted human left ventricles (17). Furthermore, improved understanding of focal cardiac mechanics may facilitate tissue engineering-based myocardial replacement therapy (18). Current cardiac functional assessments encounter limitations. While ejection fraction (EF) for global contractile function provides incomplete and often delayed cardiac mechanics of chemotherapy-induced cardiac injury $(10,12)$, strain imaging for local contractile function is limited by the 
angle dependency and significant noise of tissue Doppler-based strain assessment, and out-of-plane motion of speckle tracking (19). Pulsed-wave Doppler-acquired signals of E/A ratios from early (E-wave) and late diastolic (A-wave) ventricular inflow velocities measure only global relaxation function and are subject to variability depending on ultrasound beam angulation (13).

In this context, we developed 4D myocardial displacement quantification for analysis of segmental deformation in response to doxorubicin-induced cardiac injury and in relation to activation of the developmental Notch pathway (20). We applied displacement analysis of myocardial mechanical deformation (DIAMOND), which determines time-dependent changes in the displacement of myocardial mass centroids in 3D space, to a zebrafish model of doxorubicin-induced cardiac injury (13). We further compared focal DIAMOND displacement with global EF determination in Notch-mediated cardiac repair following doxorubicin treatment.

By integrating DIAMOND displacement with 4D LSFM imaging-acquired zebrafish embryonic hearts, we unravel that basal myocardial segments adjacent to the atrioventricular canal undergo the highest mechanical deformation. In addition, we reveal that these segments with the highest DIAMOND displacements are the ones prone to acute doxorubicin cardiac injury. We chemically and genetically manipulated the Notch signaling pathway and further identified the temporal sequence of doxorubicin chemotherapy-induced Notch pathway activation. Specifically, we determined how doxorubicin-mediated Notch activation initiates myocardial repair, starting from molecular activation of the pathway leading to architectural and mechanical phenotypes. We quantify proliferating cardiomyocytes preceding trabecular myocardium formation, leading to restoration of DIAMOND displacement cardiac mechanics. Thus, DIAMOND uncovers in vivo focal cardiac mechanics under physiological and pathological conditions such as anthracycline-induced cardiac injury.

\section{Results}

$4 D$ DIAMOND displacement development. We illustrate the process by which DIAMOND was developed to uncover segmental susceptibility to doxorubicin-induced cardiac injury. Following LSFM in vivo image acquisition of the zebrafish embryonic heart (Figure 1A), the true ventricular short axis was determined as a plane perpendicular to the vertical long axis and the horizontal long axis, both of which were manually identified off-line (Figure 1B). Next, the ventricle was resampled along its true short axis, creating a coordinate system (Figure 1C). The zebrafish heart is illustrated in its anatomical location, with the ventricle more anterior and slightly to the right compared with the atrium (Figure 1D). The ventricular short axis is divided into 8 equal segments constituted by even angles according to the intersection of a virtual division line (represented by as a red dotted line) spanning from the atrium to the ventricle with the center of the endocardial ventricular cavity (Figure 1D). The most basal segments (VII and VIII) were removed from further analyses, given they are immediately adjacent to the atrioventricular valve and thus contain less myocardium compared with the other segments (I-VI) and thereby constitute incomplete myocardial segmental territories (Figure 1E). A 3D depiction of the identified segments is illustrated, with representation of the $x, y$, and $z$ axes (Figure 1F). The ventricular resampling process was performed both at end-systole $\left(H_{S}\right)$ and at end-diastole $\left(H_{D}\right)$, leading to the corresponding but distinct end-systolic and end-diastolic matrixes (Figure $1 G$ ).

In order to use a single matrix for analyses of the resampled ventricle, a registration process was applied whereby the end-systolic matrix was systematically chosen as the reference matrix (Figure $1 \mathrm{H}$ ). To determine the transformation matrix from the end-diastolic matrix to the end-systolic matrix, a set of 3 parallelepipeds were virtually added to the framework with a known, fixed 3D geometrical rapport to the heart (Figure $1 \mathrm{H}$ ). The left upper box illustrates in 3D the partial overlap of the parallelepipeds at end-systole (green) and at end-diastole (red) due to cardiac motion (Figure 1H). Following determination and application of the transformation matrix $(T m)$, both the parallelepipeds at end-systole and at end-diastole are now rigidly aligned in the end-systole reference matrix in the upper-right box (green) (Figure $1 \mathrm{H})$. Subsequently, the transformation matrix was applied to the ventricle at end-diastole, leading to the resampled ventricle both at end-systole and at end-diastole to now be registered in the same matrix (Figure 1I). This process permits subsequent tracking in $3 \mathrm{D}$ space of the displacement vectors of mass centroids from any segment of the ventricle during the cardiac cycle (Figure 1J and Supplemental Video 1; supplemental material available online with this article; https://doi.org/10.1172/jci.insight.125362DS1). A schematic illustrates the DIAMOND displacement of ventricular segments I-VI during multiple time points in the cardiac cycle (Figure $1 \mathrm{~K}$ ), which can be simplified for quantitative analysis to 2 time points ranging from end-systole to end-diastole (Figure 1L). 
A

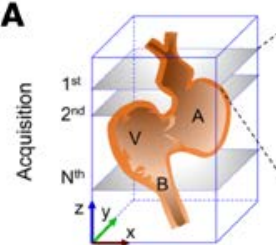

D

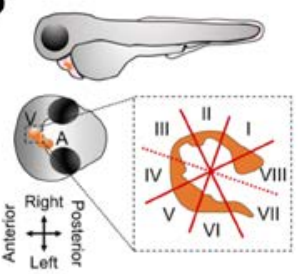

G

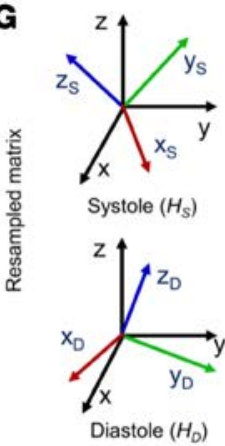

J

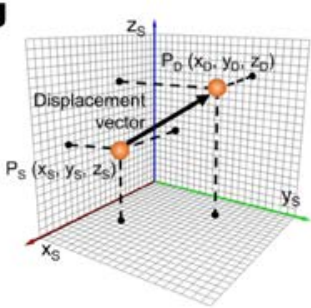

B
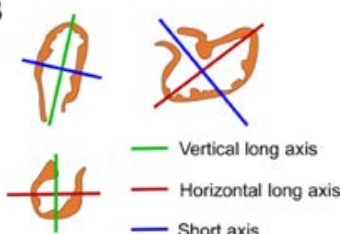

- Short axis

E

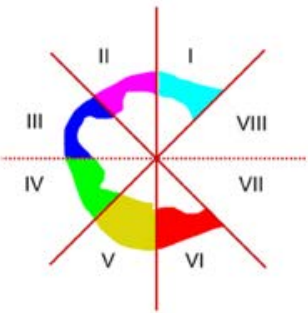

H

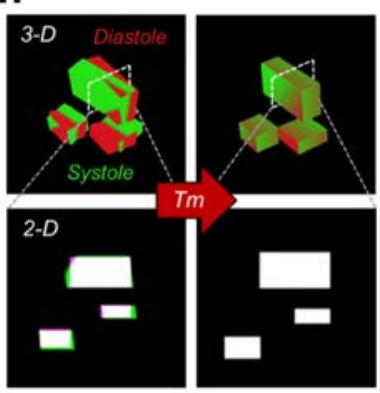

K

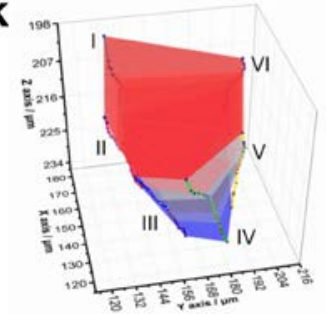

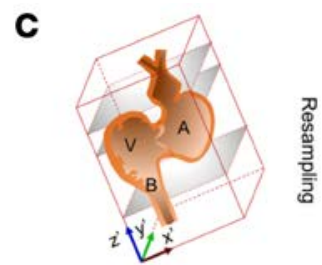

$\mathbf{F}$
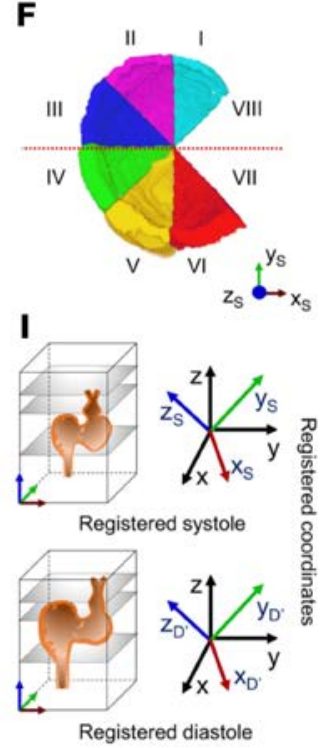

$\mathbf{L}$

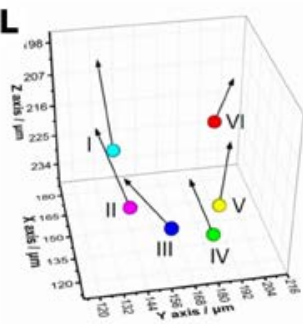

Figure 1. DIAMOND displacement analysis of focal myocardial mechanical deformation. Raw images were captured by light-sheet fluorescent microscopy (LSFM) (A) to reconstruct the digital 3D myocardium and resampled along the true anatomic short axis of the ventricle (B and $\mathbf{C}$ ). The ventricle in the schematic illustration (D) is divided into 8 segments and delineated into 6 segments with exclusion of the atrioventricular valve area (E, 2D; and $\mathbf{F}, 3 D$ ) due to relative paucity of myocardial tissue. A reference matrix from the initial resampled condition in systole was used for the reorientation of subsequent matrices (C) and registration of all the resampled slices. A group of rectangular parallelepipeds was used for the generation of transformation matrix $(T m)(\mathbf{H})$ from end-diastole to end-systole. The diastolic and systolic myocardium in the same global coordinate system were well registered by applying $T m$ to diastole (I). 3D myocardial displacements of a single segment (J) is illustrated from end-systole to end-diastole in a control myocardium. 3D displacement vectors of sequential consecutive deformations (K) and end-to-end displacement of 6 myocardial segments from end-systole to end-diastole are depicted by DIAMOND (L).

DIAMOND uncovers segmental susceptibility to doxorubicin-induced myocardial injury. Following a one-time 24-hour treatment of zebrafish with doxorubicin from day 3 to 4 after fertilization (dpf) (Figure 2A), changes in myocardial thickness from end-systole to end-diastole were observed (Supplemental Figure 1). We assessed DIAMOND displacement of ventricular segments in response to doxorubicin treatment (Figure 2B) and 48 hours later (Figure 2C). All DIAMOND figures followed the same graphical pattern as the resampled ventricles along the true short axis and the segmental divisions as illustrated above (Figure 1F). The sum vector was defined as the L2-norm and illustrated as the total height of each bar, with their weighted contributions instead of their absolute values, from the $x$ (green), $y$ (blue), and $z$ components (orange) represented within each bar. Our results indicate that under control conditions, the basal segments I and VI undergo the largest displacements (6.6\%) compared with the remaining segments II-V (3.8\%-5.1\%) (Figure 2, B and C). Com- 
A

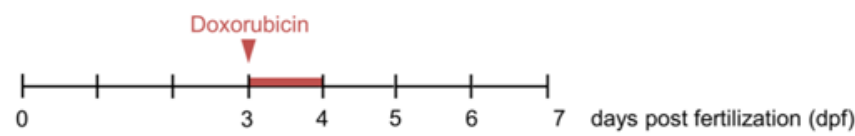

B

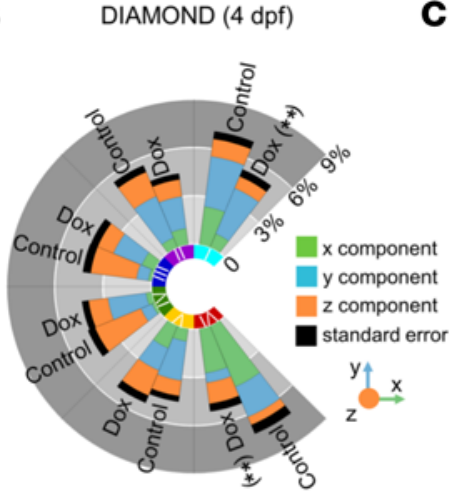

C DIAMOND (6 dpf)

D

Strain (4 dpf)

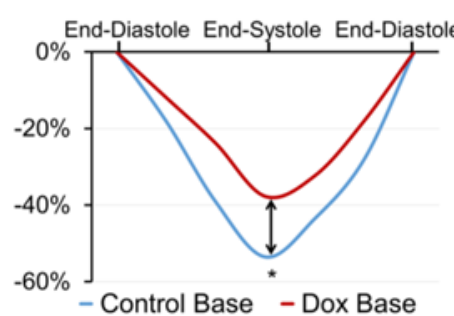

$\mathbf{F}$

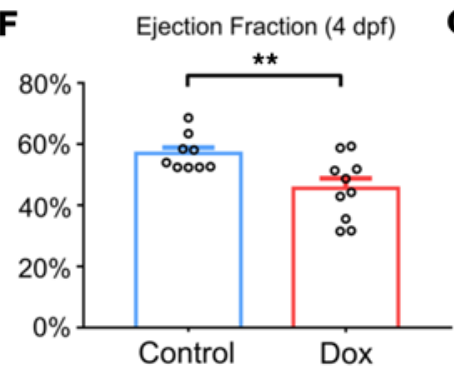

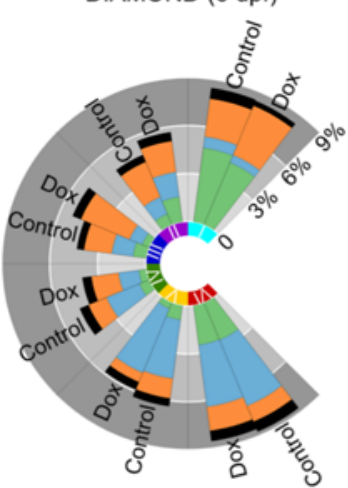

E

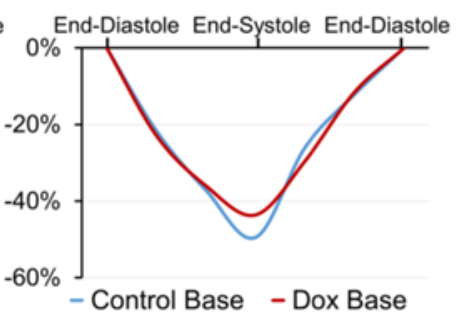

G

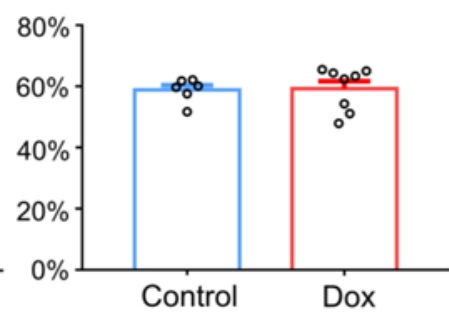

Figure 2. DIAMOND unravels new spatial insights into cardiac injury and regeneration in response to chemotherapy. (A) Experimental schedule of doxorubicin-induced myocardial injury. (B and C) Segmental comparison of DIAMOND displacement vectors between control and doxorubicin-treated groups at 4 and $6 \mathrm{dpf}$, demonstrating an acute decrease in cardiac function confined to the basal segments I and VI at $4 \mathrm{dpf}$ and restoration of function at 6 dpf ( $t$ tests, ${ }^{* *} P<0.01, n=8-10$ per group). ( $\boldsymbol{D}$ and $\mathbf{E})$ Assessment of strain in the ventricular base depicting worsening in response to doxorubicin at $4 \mathrm{dpf}$ followed by recovery at $6 \mathrm{dpf}\left({ }^{*} P<0.05, n=\right.$ 6-8 per group). ( $\mathbf{F}$ and $\mathbf{C})$ Ejection fraction attenuation in response to doxorubicin at 4 and $6 \mathrm{dpf}$, demonstrating a similar pattern as DIAMOND displacements and strain in the basal ventricular segments at the global contractile level (2-sided $t$ tests, ${ }^{* *} P<0.01, n$ = 6-10 per group).

parison with the doxorubicin-treated animals revealed that only the basal segments I and VI undergoing the largest DIAMOND displacements were susceptible to chemotherapy-induced cardiac injury (29\% decrease from $6.6 \%$ to $4.7 \%, n=10$ control and $n=8$ doxorubicin, $P<0.01$ ), whereas segments II-V with more modest DIAMOND displacements did not exhibit such changes (Figure 2B). Forty-eight hours following doxorubicin treatment ( $6 \mathrm{dpf}$ ), basal segments I and VI recovered DIAMOND displacements to control levels, suggesting focal mechanical recovery ( $n=10$ control and $n=8$ doxorubicin) (Figure 2C). In parallel, we observed a worsening in $2 \mathrm{D}$ basal strain from $-53 \%$ to $-38 \%$ in response to doxorubicin treatment $(n=$ 7 control, $n=7$ doxorubicin, $P<0.05$ ) that returned to normal 48 hours following treatment $(n=8$ control, $n=6$ doxorubicin). Assessment of conventional ventricular EF (Figure 2, D and E) demonstrated a parallel decrease in global cardiac mechanics in the setting of doxorubicin treatment (Figure $2 \mathrm{~F}, n=9$ control and $n=10$ doxorubicin, $P<0.01$, and Supplemental Videos 2 and 3, where different colors indicate maximal [green] and minimal [blue] fluorescence intensities), with recovery 48 hours after chemotherapy (Figure 2G, $n=6$ control and $n=8$ doxorubicin, and Supplemental Videos 4 and 5). Comparison of DIAMOND with EF revealed that under baseline conditions, basal segments I and VI underwent the highest displacement, and that the heightened susceptibility of these basal segments to chemotherapy-induced dysfunction (Figure 2B) was sufficient to induce a decrease in EF (Figure 2D). Therefore, our findings uncover the heterogeneity of segmental myocardial contribution to global EF, with segments undergoing the most significant DIAMOND displacement exhibiting the highest propensity for doxorubicin-induced myocardial injury. 
Doxorubicin treatment and Notch activation. Prior to determining a putative role of the Notch pathway in global and focal myocardial function following chemotherapy, we assessed the temporal Notch activation under 3 conditions: control, doxorubicin, and doxorubicin combined with Notch inhibition ( $\gamma$-secretase inhibitor [DAPT]) conditions (Figure 3A). We crossed a cmlc2:mCherry transgenic line (Figure 3, B-D) with the Tp1-gfp Notch activation reporter line (Figure 3, E-G), to generate dual fluorescence images with $\mathrm{Tg}$ (cmlc2:mCherry;tp1:gfp) (Figure 3, H-J), thereby permitting tracking of cardiac Notch activation. Notch expression was normalized to total myocardial volumes (Figure $3 \mathrm{~K}$ ). Our results indicate that following doxorubicin treatment ( $4 \mathrm{dpf}$ ), there was a significant increase from $19 \%$ to $38 \%$ in Notch expression $(n=9$ control, $n=9$ doxorubicin, $P<0.01$ ) which was attenuated in response to concomitant $\gamma$-secretase inhibitor DAPT ( $n=10$ doxorubicin + DAPT, $P<0.01$ vs. doxorubicin, $P$ not significant vs. control). Twenty-four hours following doxorubicin treatment $(5 \mathrm{dpf})$, there was a decrease in relative Notch activity $(n=9$ control, $n=9$ doxorubicin, $n=10$ doxorubicin + DAPT), which remained higher than control levels (33\% vs. $27 \%$, respectively, $P<0.01$ ). Forty-eight hours after doxorubicin treatment (6 dpf), Notch expression fell below control levels $(22 \%$ vs. $30 \%$, respectively, $P<0.01)$ to values similar to those in the doxorubicin + DAPT treatment group ( $n=8$ control, $n=9$ doxorubicin, $n=10$ doxorubicin + DAPT). Segmental analysis using the $\mathrm{Tp} 1$ reporter line illustrated focal variation of Notch activation at $4 \mathrm{dpf}$ (Supplemental Figure 2). Taken together, these results indicate an acute increase in cardiac Notch activation in response to doxorubicin treatment, which progressively decreased following the acute chemotherapy injury process.

Doxorubicin treatment and cardiomyocyte proliferation. To assess the cellular effects of molecular Notch signaling under control, doxorubicin, and doxorubicin combined with Notch inhibition (DAPT) conditions (Figure 4A), we used the fluorescent ubiquitylation-based cell cycle indicator (FUCCI) transgenic line to localize proliferating $\left(\mathrm{red}^{-}\right.$green $\left.^{+}\right)$and quiescent $\left(\mathrm{red}^{+}\right.$green $^{-}$and red $^{+}$green $^{+}$) cardiomyocytes (Figure 4, B-D). We demonstrated an initial increase in the ratio of proliferating to non-proliferating cardiomyocytes from $7.4 \%$ in controls to $8.8 \%$ in doxorubicin-treated animals ( $n=8$ control, $n=7$ doxorubicin) at the end of chemotherapy exposure ( $4 \mathrm{dpf})$ that became significant 24 hours after chemotherapy (5 dpf) $(8.3 \%$ in controls, $11.5 \%$ in doxorubicin, $P<0.05, n=8$ control, $n=7$ doxorubicin), with a return to control levels 48 hours after chemotherapy ( 6 dpf). Reciprocally, doxorubicin treatment combined with Notch inhibition using DAPT resulted in a persistently low ratio of proliferating cardiomyocytes as compared with control levels at all time points. Segmental cardiomyocyte proliferation was further assessed at $5 \mathrm{dpf}$, with the lowest levels observed in segment 1 (Supplemental Figure 3). Combined, these findings support the Notch-dependent increase in cardiomyocyte proliferation 24 hours after doxorubicin chemotherapy.

Cardiac trabeculation following doxorubicin treatment and Notch pathway modulation. We quantified the effect of doxorubicin-mediated Notch activation on cardiac trabeculation in response to (i) the inhibitor DAPT, and (ii) rescue by downstream effectors (Notch intracellular domain [NICD] mRNA and neuregulin-1 [NRG1] mRNA) (Figure 5A). Compact (blue, Figure 5B) and trabeculated (red, Figure 5C) myocardium were delineated in serial 2D images (Figure 5D) and quantified in the $3 \mathrm{D}$ myocardium using different treatment permutations (Figure 5, E-M). Our results indicate that the degree of trabeculated myocardium increases in control animals from $5.6 \%$ at $4 \mathrm{dpf}(n=10)$ (Figure $5 \mathrm{~N})$ to $8.9 \%$ at $6 \mathrm{dpf}(n=7, P<0.01)$ (Figure 5O) in the setting of ongoing cardiac morphological maturation. Under control conditions, overexpression of NRG1 but not of NICD further increased the trabecular volume at both 4 and $6 \mathrm{dpf}$.

The myocardial trabecular volume decreased at $4 \mathrm{dpf}$ from $5.6 \%$ to $3.6 \%$ following doxorubicin-alone treatment ( $n=10$ control, $n=8$ doxorubicin, $P<0.01$ ) (Figure 5, E, F, and $\mathrm{N}$ ). There was a similar decrease in the setting of concomitant doxorubicin and DAPT treatment (sham groups in Figure $5 \mathrm{~N})(n=8$ doxorubicin + DAPT, $P<0.01$ vs. control). Forty-eight hours after completion of chemotherapy ( $6 \mathrm{dpf}$ ), the myocardial trabecular volume recovered in the doxorubicin-alone treatment group $(n=7$ control, $n=8$ doxorubicin), but not in the setting of concomitant DAPT exposure $(n=7$ doxorubicin + DAPT, $P<0.05$ vs. control, $P<0.01$ vs. doxorubicin) (sham groups in Figure 5O).

Following doxorubicin-alone treatment, overexpression of either NICD or NRG1 rescued the decrease in trabeculation at $4 \mathrm{dpf}$ (Figure $5 \mathrm{~N})(n=6$ and $P<0.01$ for both conditions vs. doxorubicin sham). At 6 $\mathrm{dpf}$, when trabeculation had already been restored in the doxorubicin-alone treatment group (sham), i.e., architectural recovery had occurred, there was no added benefit of NICD or NRG1 overexpression (Figure 5O). On the other hand, following the "two-hit" injury process of doxorubicin exposure and Notch inhibition by DAPT, which precluded recovery of trabeculation at $6 \mathrm{dpf}$ (sham condition), there was an increase in trabeculation with NRG1 or NICD (compared with doxorubicin + DAPT sham) to control levels 


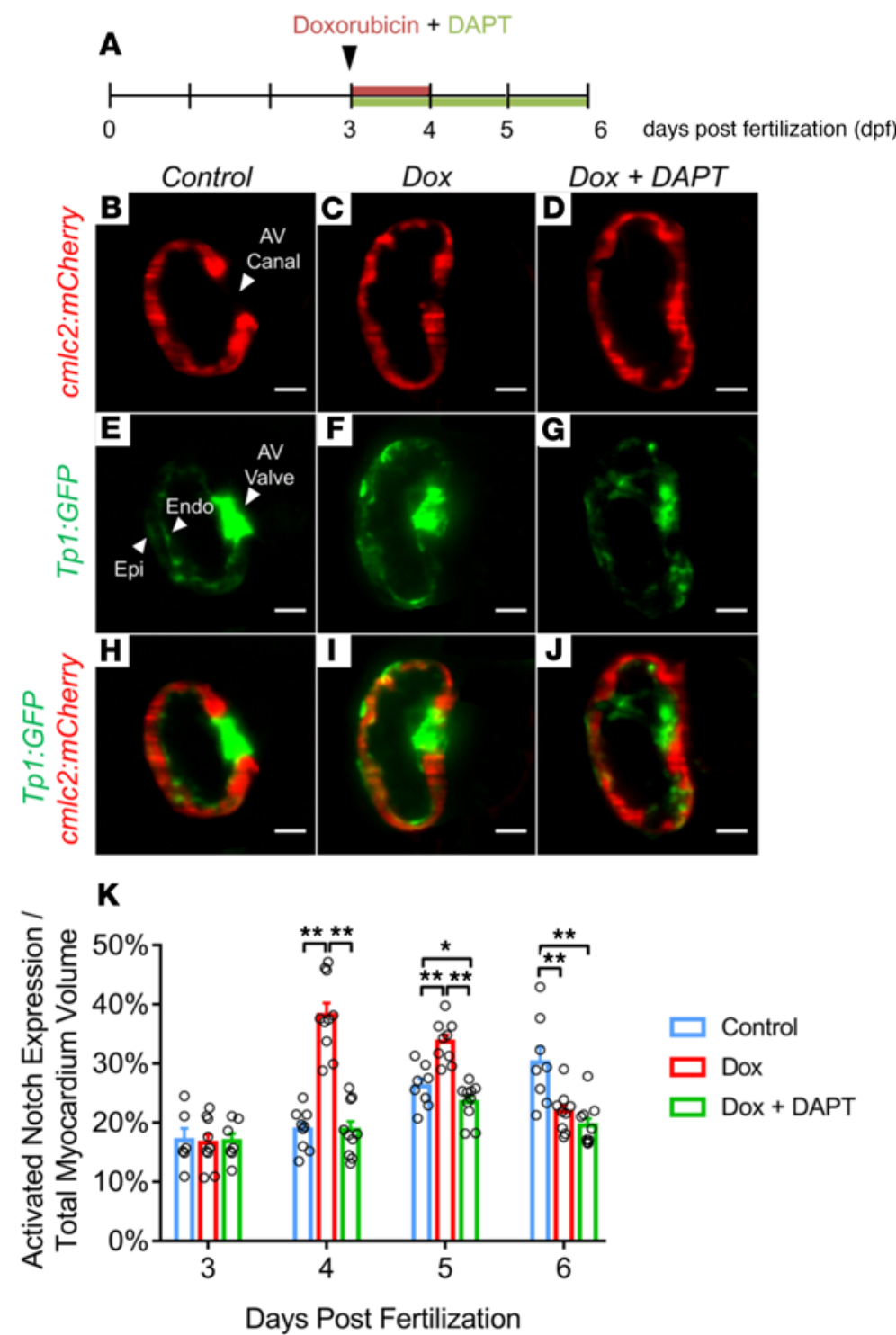

Figure 3. Chemo-induced injury induces cardiac Notch activation. (A) Experimental schedule using a $\mathrm{Tg}(\mathrm{cm} / \mathrm{c} 2$ : $\mathrm{mCher}$ $r y ; T p 1: g f p)$ line. (B-D) The cmlc2:mCherry transgene was used to delineate the myocardium. (E) The Tp1gfp signal was demonstrated in both the endocardium (Endo) and the epicardium (Epi). AV, atrioventricular. (F) Following 24-hour doxorubicin treatment, a robust increase in TP1 signal indicated Notch activity throughout the ventricle. (C) The increase in Notch activity was attenuated when embryos were cotreated with the Notch $\gamma$-secretase inhibitor DAPT. (H-J) $g f p$ and mCherry channels were merged. (K) Quantitative analysis of Tp1-gfp signal volume was normalized to the total myocardium volume (ANOVA, ${ }^{*} P<0.05$, ${ }^{*} P<0.01, n=8-10$ per group). Signals emanating from the atrioventricular valve were excluded from analyses. Scale bars: $25 \mu \mathrm{m}$.

observed at $4 \mathrm{dpf}($ Figure $5 \mathrm{~N})(n=7$ doxorubicin $+\mathrm{DAPT}+\mathrm{NICD}, n=6$ doxorubicin $+\mathrm{DAPT}+N R G 1)$ that was maintained at $6 \mathrm{dpf}$ (Figure 5O) $(n=6$ doxorubicin + DAPT + NICD, $n=5$ doxorubicin + DAPT $+N R G 1, P<0.05$ vs. doxorubicin + DAPT for both). The above architectural findings at 48 hours after chemotherapy persisted at 72 hours after chemotherapy ( $7 \mathrm{dpf}$ ), suggesting completion of the cardiac repair process (Supplemental Figure 4). Taken together, our results demonstrate that the ratio of trabeculated to total myocardium decreases in response to acute doxorubicin treatment, recovers 48 hours thereafter, and that this recovery is mediated by Notch signaling.

DIAMOND assessment of segmental cardiac mechanics in doxorubicin-induced cardiac injury and Notch pathwaymediated repair. We next applied DIAMOND assessment of segmental cardiac mechanics following doxorubicin treatment under conditions of Notch pathway modulation using the inhibitor DAPT and rescue by the downstream effectors NICD and NRG1 mRNA (Figure 6A) during acute chemotherapy injury (Figure 6B) and recovery (Figure 6C). We initially demonstrated the heterogeneity of segmental myocardial contribution to global EF in zebrafish, with basal segments I and VI undergoing the most significant DIAMOND displacement exhibiting the highest propensity for doxorubicin-induced injury (Figure 2). By combining doxorubicin treatment with Notch pathway inhibition using DAPT, we observed a homogeneous decrease in DIAMOND displacement involving all myocardial segments $(n=10$ control, $n=6$ doxorubicin + DAPT, $P<0.05$ ) (Figure $6 \mathrm{~B})$. Importantly, 48 hours after doxorubicin treatment and with DAPT cotreatment, all myocardial segments had recovered DIAMOND displacement, save the basal segments I and VI exhibiting the highest displacement under control 
A

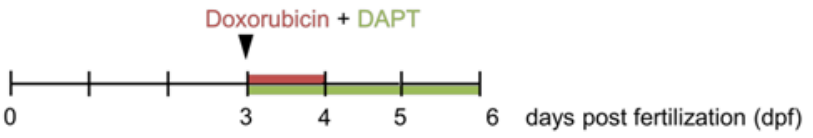
Control Dox

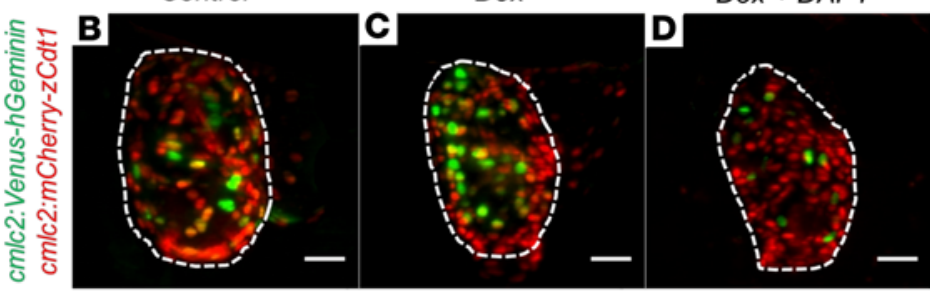

\section{E}

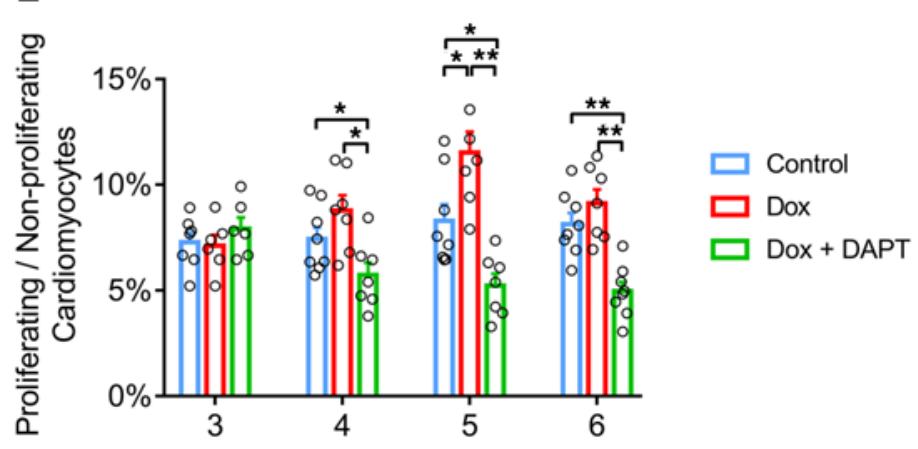

Figure 4. Cardiomyocyte proliferation following chemo-induced injury. (A) Experimental schedule using the fluorescent ubiquitylation-based cell cycle indicator (FUCCI) line. (B-D) Representative maximum intensity projection images at $5 \mathrm{dpf}$, uncovering proliferating (cm/c2:Venus-hGeminin, green) and nonproliferating (cm/c2:mCherry-zCdt1, red/orange) cardiomyocytes. The contour of the ventricle is delineated by white dashed lines. Proliferating cardiomyocytes increased significantly following doxorubicin treatment, whereas cotreatment with DAPT significantly decreased cardiomyocyte proliferation. (E) Quantitative analysis of percentages of proliferating cardiomyocytes (ANOVA, ${ }^{*} P<0.05,{ }^{* *} P<0.01, n$ = 7-10 per group). Scale bars: $25 \mu \mathrm{m}$.

Days Post Fertilization

conditions, which remained abnormal ( $n=10$ control, $n=7$ doxorubicin + DAPT, $P<0.05)$. Further, we demonstrate that rescue with the Notch downstream effectors NICD and NRG1 restores DIAMOND displacement in all segments, during both acute doxorubicin injury and Notch inhibition with DAPT (Figure 6B) and during recovery (Figure $6 \mathrm{C}$ ) ( $n=6$ for both conditions and at both time points). Percent cell shortening was also measured to assess cardiomyocyte single cell contraction and relaxation (21). Our results indicate a decrease in cell shortening with doxorubicin treatment with or without DAPT, and a rescue in single cell cardiac mechanical function following NRG1 overexpression (Supplemental Figure 5). We additionally manipulated the time course of exposure to DAPT-mediated Notch inhibition following doxorubicin treatment (Supplemental Figure 6). DAPT treatment at 24 and 48 hours after doxorubicin exposure allowed for a time delay in the inhibition of Notch signaling and impaired the restoration of segmental cardiac function only in segment I, which was also the segment with the lowest activation of Notch signaling (Supplemental Figure 2) and focal cardiomyocyte proliferation (Supplemental Figure 3) following chemotherapy injury.

We also assessed the effect on global EF of doxorubicin treatment combined with Notch modulation (Figure $6, \mathrm{D}$ and E). NICD or NRG1 mRNA exposure slightly increased EF in control animals at both 4 $\operatorname{dpf}(n=9$ control, $n=6$ NICD, $n=6$ NRG1, $P<0.05$ for both vs. control) and at 6 dpf ( $n=6$ control, $n=$ 6 NICD, $n=5$ NRG1, $P<0.05$ for both vs. control). The decrease in EF following doxorubicin treatment was rescued by Notch downstream effectors during acute chemotherapy injury ( $n=10$ doxorubicin, $n=6$ doxorubicin + NICD mRNA, $n=6$ doxorubicin + NRG1 mRNA, $P<0.01$ for both vs. doxorubicin), and EF remained normal 48 hours after chemotherapy under the same conditions $(n=8$ doxorubicin, $n=6$ doxorubicin + NICD mRNA, $n=5$ doxorubicin + NRG1 mRNA). Combining doxorubicin with Notch inhibition using DAPT led to a decrease in EF both during acute chemotherapy injury ( $n=11$ doxorubicin + DAPT, $P<0.01$ vs. control) and 48 hours thereafter $(n=8$ doxorubicin + DAPT, $P<0.01$ vs. control) that was rescued by the Notch downstream effectors NICD mRNA $(n=6$ doxorubicin + DAPT + NICD mRNA, $P<0.01$ vs. doxorubicin + DAPT at $4 \mathrm{dpf}$ and $P<0.05$ at $6 \mathrm{dpf}$ ) and NRG1 mRNA ( $n=6$ doxorubicin + DAPT + NRG1 mRNA, $P<0.01$ vs. doxorubicin + DAPT at both 4 and $6 \mathrm{dpf})$. The above functional findings at 48 hours after chemotherapy persisted at 72 hours after chemotherapy ( $7 \mathrm{dpf})$, indicating completion of the cardiac repair process (Supplemental Figure 7). Therefore, we demonstrate that whereas only basal segments I and VI, which exhibit the highest displacements, are susceptible to doxorubicin inju- 

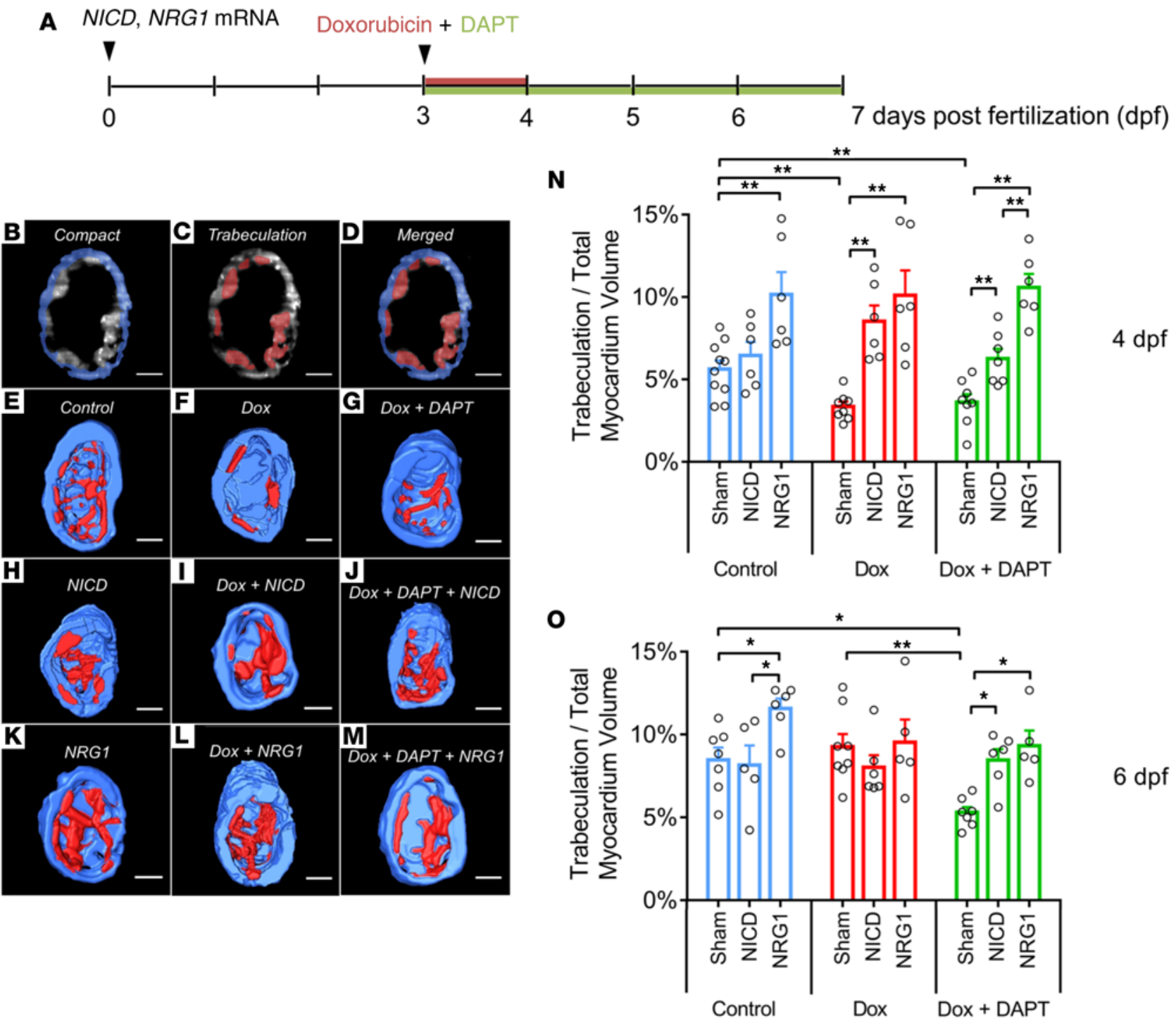

Figure 5. The 3D cardiac trabecular architecture in response to doxorubicin-induced injury and genetic manipulation. (A) Diagram depicting the experimental schedule. (B-D) Light-sheet imaging-acquired 3D trabecular network for quantitative analyses. Compact myocardium (blue) and trabeculated myocardium (red) were manually segmented. (E-G) Representative 3D reconstructed images reveal a significant reduction of the trabecular network in the groups treated with doxorubicin and doxorubicin $+\gamma$-secretase inhibitor (DAPT) at $4 \mathrm{dpf}$. (H-M) Microinjection of the Notch downstream effectors Notch intracellular domain (NICD) and neuregulin-1 (NRG1) mRNA rescued the DAPT-attenuated trabecular network at $4 \mathrm{dpf}$ ( $\mathbf{N}$ and $\mathbf{0}$ ) Quantitative analysis of trabecular volume normalized to total myocardium volume at 4 and $6 \mathrm{dpf}$ (ANOVA, ${ }^{*} P<0.05,{ }^{* *} P<0.01, n=5-10$ per group). Scale bars: $25 \mu \mathrm{m}$.

ry in the acute chemotherapy setting, superimposing Notch pathway inhibition leads to a global decrease in DIAMOND displacements (Figure 6B). All myocardial segments exposed to doxorubicin and Notch inhibition recover 48 hours thereafter, save basal segments I and VI (Figure 6C), with associated continued decreased EF (Figure 6E), a phenomenon rescued by Notch downstream effectors.

Temporal sequence of doxorubicin-induced cardiac injury, Notch-mediated recovery, and normalization of cardiac mechanics. We present a schematic diagram summarizing our experimental procedures and findings (Figure 7). Following 24 hours treatment with doxorubicin from 3 to $4 \mathrm{dpf}$, we observed a decrease in myocardial function assessed by DIAMOND displacement and conventional EF at $4 \mathrm{dpf}$. In parallel, there was an increase in cardiac Notch expression in the doxorubicin-treated group at $4 \mathrm{dpf}$, followed by an increase in cardiomyocyte proliferation at $5 \mathrm{dpf}$, and a return of the cardiac architecture to normal trabecular versus compact ratios at $6 \mathrm{dpf}$. Concomitantly at $6 \mathrm{dpf}$, we observed a functional recovery in cardiac mechanical parameters assessed focally by DIAMOND and globally by EF that was maintained at $7 \mathrm{dpf}$. 

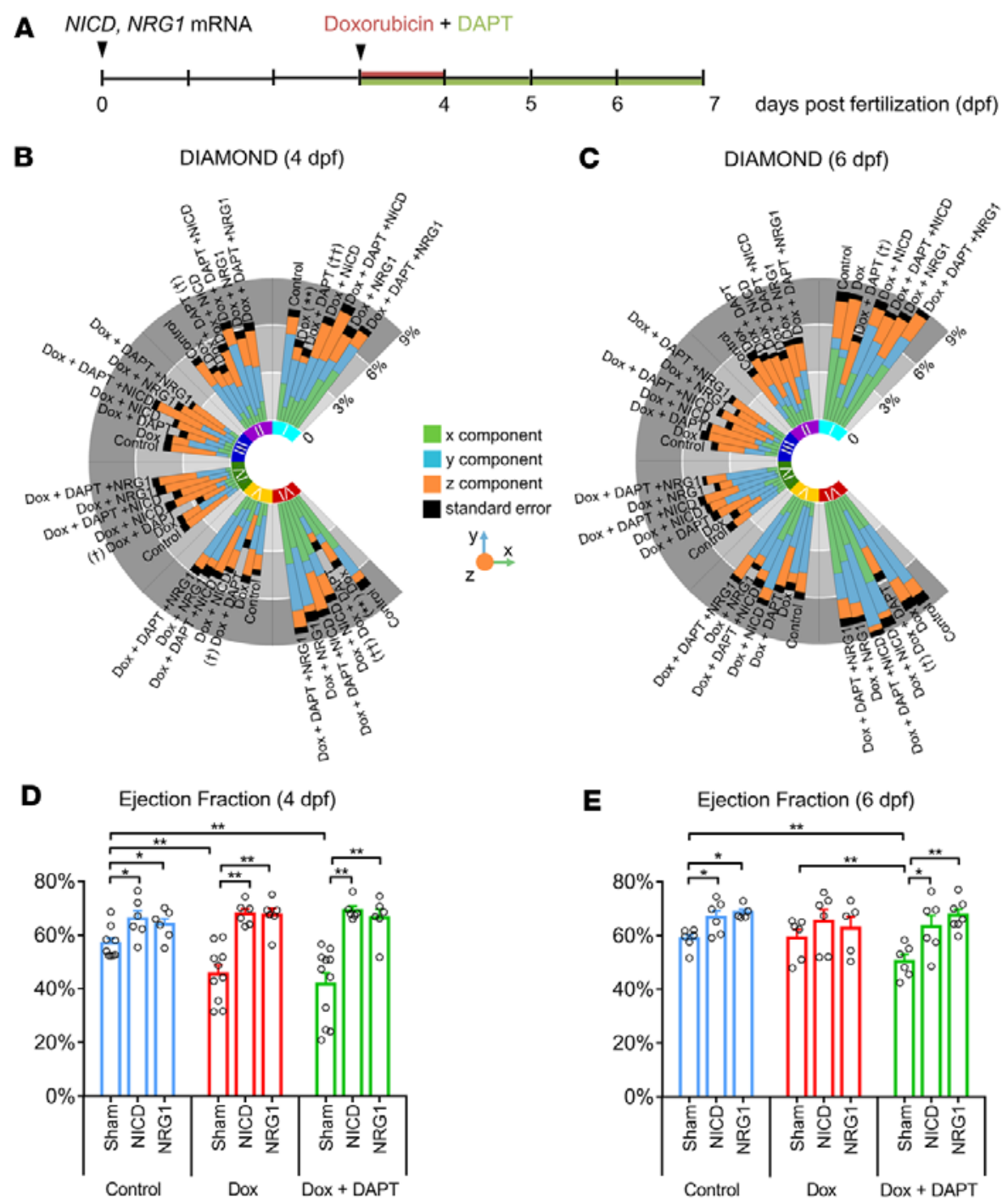

Figure 6. DIAMOND mechanics for segmental localization and quantification of NICD and NRG1 mRNA-mediated myocardial protection following doxorubicin-induced injury. (A) Experimental schedule. (B and C) NICD and NRG1 mRNA microinjection rescued the reduction of myocardial displacement in segments I and VI by doxorubicin and $\gamma$-secretase inhibitor (DAPT) at $4 \mathrm{dpf}$. At $6 \mathrm{dpf}$, the inhibition of Notch signaling by DAPT impaired the restoration of segmental cardiac function ( ${ }^{* *} P<0.01$ Dox vs. control; $\uparrow P<0.05, \uparrow \uparrow P<0.01$, Dox + DAPT vs. control, $n=6-10$ per group). ( $\mathbf{D}$ and $\mathbf{E})$ Ejection fraction demonstrates at the global level that NICD and NRG1 mRNA micro-injection confers cardiac protection from doxorubicin-induced injury (ANOVA, ${ }^{*} P<0.05,{ }^{* *} P<0.01, n=5-11$ per group).

\section{Discussion}

A rigorous strategy for quantitative analysis of focal myocardial function is paramount to characterize cardiac function under chemotherapy-induced injury and cardiomyopathy beyond traditional EF, known to be an insensitive indicator of cardiomyocyte injury and regenerative ability $(1,2)$. Measurement of $2 \mathrm{D}$ displacement in humans by echocardiography has been previously proposed; however, this method is an oversimplification of 3D myocardial deformation, in addition to being limited by signal-to-noise ratio and subjectivity of assessment (22). In the current study, we describe the development and application of DIAMOND, a method for in vivo tracking of 3D focal cardiac mechanics that we validated with strain imaging as an independent standard of reference. We present a systematic algorithm to determine DIAMOND displacement; our method, written in MATLAB language, is available as an open-source file for potential adoption and determination of reproducibility by other laboratories. Its integration with 4D LSFM imaging provides new spatial insights into zebrafish cardiac mechanics, unraveling a segmentally varied dis- 


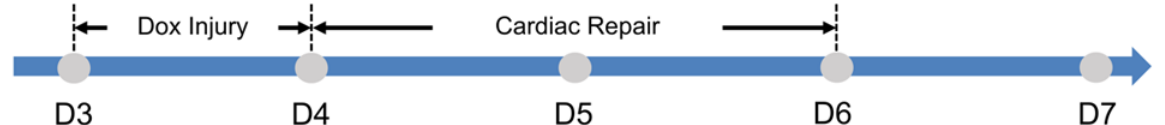

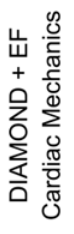
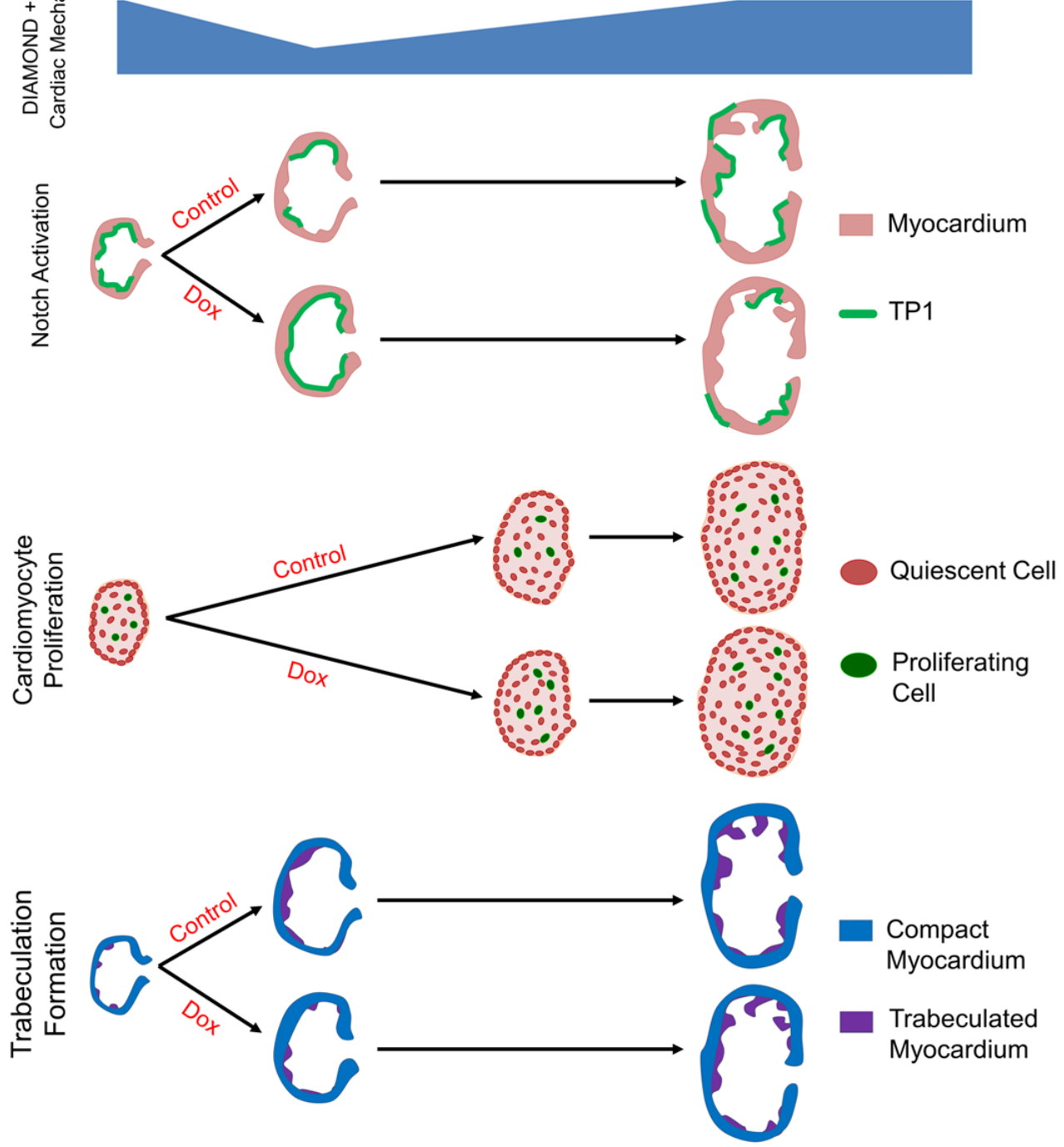

Figure 7. Summary diagram depicting the temporal sequence of cardiac architectural and mechanical regeneration after doxorubicin-induced injury. At 4 dpf (D4), 24-hour treatment with doxorubicin (Dox) precipitates an attenuation of trabeculation development, resulting in an acute decrease in cardiac function demonstrated by DIAMOND at the segmental level and ejection fraction at the global level. In parallel, a robust upregulation of Notch signaling is observed at 4 $\mathrm{dpf}$, which precedes the regeneration process indicated by an increase in proliferating cardiomyocytes peaking at $5 \mathrm{dpf}$. At $6 \mathrm{dpf}$, the trabecular network and cardiac mechanical function assessed by DIAMOND and EF are fully restored, an observation further confirmed at $7 \mathrm{dpf}$.

placement and susceptibility pattern to doxorubicin-induced injury and regeneration. To further investigate the biomechanical mechanism underlying the restoration of myocardial function after injury demonstrated by DIAMOND, we manipulated the Notch pathway to demonstrate a temporal sequence of Notch-mediated cardiac repair. Thus, this methodology is anticipated to facilitate and provide mechanistic insights into in vivo cardiac function in a high-throughput manner using zebrafish embryos (23).

The zebrafish was selected as the in vivo model in this study due to its cardiac regenerative capacity, optical transparency during development, and genetically tractable system $(4,6,7,9)$. We integrated 4D LSFM with a post-imaging synchronization algorithm $(11,12)$ to acquire high-quality $3 \mathrm{D}$ volume rendering of the contracting heart. Other imaging modalities, such as confocal microscopy, are limited by their intrinsic depth penetration, axial resolution, and long scanning time. More clinically oriented imaging techniques, such as micro-CT, micro-MRI, and micro-PET, do not allow tracking of fluorescently labeled proteins with high spatial resolution in the embryonic zebrafish beating heart (24). 3D strain imaging is gaining increasing traction (25); however, it is not currently feasible in smaller experimental animals such as zebrafish.

To assess ventricular function in response to doxorubicin treatment in adult zebrafish, we previously synchronized micro-electrocardiogram ( $\mu \mathrm{ECG}$ ) signals with blood flow velocities in beating hearts using 
a high-frequency 30-MHz ultrasound array (13). Pulsed-wave Doppler-acquired signals of (i) E/A ratios of ventricular inflow velocities to measure global relaxation function and (ii) the myocardial performance index as an integrated measure of both systolic and diastolic function were obtained. Doxorubicin-injected adult zebrafish developed ventricular diastolic dysfunction and worsening global cardiac function on day 30 , followed by normalization on day 60 (13). Whereas these studies in adult zebrafish permitted functional characterization of global cardiac mechanics following chemotherapy, our present work with DIAMOND permits segmental characterization of cardiac function and tracking of fluorescently labeled structures. Therefore, 4D LSFM, with its high spatial and temporal resolution, deep imaging depth, and low phototoxicity, provides a basis for 3D displacement analysis of the segmental ventricle in zebrafish embryos, not otherwise readily feasible with other imaging modalities (Supplemental Table 1) (23).

By localizing the horizontal and vertical long axes of the ventricle, as well as exploiting the atrioventricular canal as an anatomical landmark, DIAMOND, for the first time to our knowledge, established a methodology for tracking focal cardiac function in $3 \mathrm{D}$, permitting quantitative assessment. Furthermore, to maintain a rigorous correspondence of the segments as well as the spatial relationship between systole and diastole, we built in a $3 \mathrm{D}$ rigid registration to restore any mismatch of the matrices due to the resampling process. We demonstrated different information provided by global EF and focal DIAMOND displacement, with basal segments adjacent to the atrioventricular canal displaying the highest 3D displacement and being the most susceptible to chemotherapy-induced cardiac injury. Importantly, DIAMOND is able to capture additional spatial information to localize segmental mechanical function and provide insights into local doxorubicin-induced myocardial injury and regeneration. Specifically, the higher displacement and the higher susceptibility to doxorubicin-induced injury in the basal segments provide a means to uncover variations in local myofibril architecture or metabolic activity during both development and regeneration.

The mechanism of increased susceptibility of the most dynamic myocardial segments to doxorubicin is presently unknown, but may involve increased metabolic activity, increased oxygen consumption, and increased reactive oxygen species generation under physiological conditions that may be further potentiated by doxorubicin toxicity (2). To scrutinize doxorubicin-induced attenuation followed by restoration of cardiac function demonstrated by DIAMOND, we studied Notch signaling-mediated regeneration. Previous studies have shown the critical role of Notch/ErbB2/NRG1 signaling in regulating cardiomyocyte proliferation and regeneration (26-28). By utilizing the combination of a Tp1 Notch reporter line (29), FUCCI cardiomyocyte proliferation monitoring line (30), and DIAMOND, we established a temporal sequence of doxorubicin-induced injury and regeneration, starting with Notch activation, and followed by cardiomyocyte proliferation, trabeculation restoration, and cardiac function normalization 48 hours after doxorubicin treatment. Moreover, DIAMOND further demonstrated the cardioprotective function of NICD and NRG1 (31), as indicated by an unaffected displacement in each segment after doxorubicin treatment. Our results establish a temporal sequence and spatial characterization of cardiac regeneration following doxorubicin injury, initiated by upregulation of Notch signaling, followed by increased cardiomyocyte proliferation, leading to restoration of the myocardial trabecular network and cardiac mechanical function assessed by DIAMOND and EF. The demonstrated cardiac regeneration cycle following chemotherapy-mediated injury, coupled with focal DIAMOND cardiac mechanics, provides what we believe to be a novel approach for high-throughput in vivo screening of drugs mitigating doxorubicin-induced toxicity, and the temporal sequence might serve as reference time points for evaluating the interplay of gene expression, cardiac architecture, and mechanics during the regeneration process.

Overall, DIAMOND provides focal cardiac mechanics assessment in zebrafish embryos to localize, track, and quantify segmental myocardial function in response to doxorubicin-induced cardiac injury and Notch signaling-mediated myocardial regeneration. Further applications of DIAMOND should include investigating the colocalization of cardiac mechanics and gene expression with fluorescent labels, and integration with micro-CT or micro-MRI suitable in larger animal models of chemotherapy-induced injury for the multiscale assessment of cardiac regeneration (32).

\section{Methods}

LSFM imaging system. The custom-made LSFM imaging system utilized a continuous-wave laser (Laserglow Technologies) as the illumination source to image all transgenic zebrafish lines $(11,12,23)$. The dual detection module was composed of 2 scientific complementary metal oxide semiconductor (sCMOS, ORCA-Flash4.0, Hamamatsu) cameras and 2 sets of filters (Semrock) for dual-channel imaging. This detection module was perpendicularly installed to the illumination plane. The sample holder was orient- 
ed by a 5-axis mounting stage for scanning of the biological specimen. Both illumination and detection modules were controlled by a computer with a dedicated solid-state drive (SSD) redundant array of independent disks level 0 (RAID 0) storage for fast data streaming. Each LSFM frame was acquired within a 20 -ms exposure time, while the resolving power in cross section was $\sim 0.65 \mu \mathrm{m}$ and the step size between consecutive frames was $\sim 2 \mu \mathrm{m}$ (Figure $1 \mathrm{~A}$ and Supplemental Table 1).

Image synchronization for assessment of cardiac mechanics. We synchronized the 4D heart to address cardiac motion using a computational algorithm as previously described $(12,14)$. Briefly, 3-4 zebrafish cardiac cycles were acquired in each slice, the period was estimated, and the same instantaneous moment in different periods was correlated. Next, 2 image sequences were aligned before 3D structure reconstruction due to the idle time between slices, and the relative shift was formulated in the form of quadratic minimization. Finally, we converted the relative shift to the absolute shift with respect to the first image sequence. Although relative shift could be used to align 2 adjacent frames, all of the frames were aligned to reconstruct the entire heart. Upon obtaining all relative shifts, a relation to align the next slice given the previous slice was built. This recurrent relation was transformed into an absolute relation by solving a linear equation using the pseudoinverse approach. Subsequently, EF and 2D strain were quantified as previously reported (12). To quantify myocardial thickness, 5 equally spaced measurements were taken (ImageJ, NIH) along the radial direction for each segment (I-VI) at end-systole and end-diastole following image synchronization and reconstruction. The average myocardial thickness of each segment at end-systole and end-diastole was calculated by computing the average of the 5 measurements. Changes in myocardial thickness were calculated as the difference between measurements in end-diastole and end-systole.

DIAMOND image resampling. For DIAMOND image resampling, we reconstructed the 3D digital heart from the individual cardiac phase in the global Cartesian coordinate system $(x, y, z)$ and segmented raw images into binary datasets in Amira 6.01 (FEI). Based on the anatomical structure, we manually reoriented the heart by localizing the atrioventricular (AV) canal and short/long axes of the heart (Figure 1B), and isotropically resampled the heart $(333 \times 333 \times 400$ voxels, $1 \times 1 \times 1 \mu \mathrm{m} /$ voxel $)$ along the short axis in a new coordinate system $\left(x^{\prime}, y^{\prime}, z^{\prime}\right)$ (Figure 1C). The resampled systole and diastole data were separately saved in different matrices. Both raw data and segmented data were processed under the same procedures. In the subsequent analysis, the coordinate system of end-systole was set as default.

DIAMOND image division. After acquiring the resampled data, we identified the virtual centerline of the ventricular and atrial cavity, which were connected to generate a baseline (dashed line in Figure 1D). The ventricle was evenly divided into 8 portions (I-VIII) along the true short axis, followed by the removal of segments VII and VIII given their relative lack of myocardial tissue in proximity to the atrioventricular valve (Figure 1E). Next, we saved the remaining six 3D segments into separate image stacks, without changing their relative positions (Figure 1F). From end-systole to end-diastole, we generated distinct global coordinate systems to account for the arbitrary orientation of the contracting heart at each time point.

DIAMOND image registration. We established displacement vectors in $3 \mathrm{D}$ space to quantify the segmental movement of each portion from end-systole to end-diastole. The resampling process along the true short axis of the ventricle led to different image matrixes at end-systole and end-diastole. We therefore registered the coordinate systems of resampled systole and diastole under the same global coordinate system $(x, y, z)$ (Figure $1 G)$. To conform to the complexity of cardiac structures and deformation from systole to diastole, we artificially created a group of rectangular parallelepipeds that were also resampled in the end-systole and end-diastole, to allow for $3 \mathrm{D}$ rigid registration that preserves the distance between 2 points and angles subscribed by 3 points. The artificial rectangular parallelepipeds were designed to be identical in shape and size in both the end-systole and end-diastole matrixes. When the end-diastole rectangular parallelepiped (red) was registered to the end-systole rectangular parallelepiped (green), the ensuing discrepant 3D location permitted the derivation of a unique matrix of rigid transformation consisting of rotation and translation from the end-diastole matrix to the end-systole matrix (Figure $1 \mathrm{H}$ ). We performed the registration and regularized energy minimization using the MATLAB Image Processing Toolbox. We demonstrated how to minimize the error function $(E)$ in the transformation from diastole to systole system by aligning 2 groups (for each of the cardiac cycle time points) of 3 rectangular parallelepipeds in the global coordinate system as follows:

$$
E=\min \left(\sum_{i=1}^{n}\left\|\operatorname{Tm}\left(p_{i}\right)-p_{i}^{\prime}\right\|^{2}\right)
$$


where $T m$ denotes the transformation matrix function from diastole to systole; and $p_{i}$ and $p_{i}{ }^{\prime}(i=1,2, \ldots$, $n)$ denote feature points of the moving (end-diastole) and fixed (end-systole) targets, respectively. The generated transformation matrix included 3D rotation and translation. The final transformation from diastole to systole was indicated as follows:

$$
\underline{p^{\prime}}=R p+T, \quad\left[\begin{array}{c}
x^{\prime} \\
y^{\prime} \\
z^{\prime} \\
1
\end{array}\right]=\left[\begin{array}{cccc}
r_{11} & r_{12} & r_{13} & t_{x} \\
r_{21} & r_{22} & r_{23} & t_{y} \\
r_{31} & r_{32} & r_{33} & t_{z} \\
0 & 0 & 0 & 1
\end{array}\right]\left[\begin{array}{l}
x \\
y \\
z \\
1
\end{array}\right]
$$

\section{Equation 2}

Where $p$ denotes the end-diastolic condition; $\underline{p}^{\prime}$ indicates the transformed systolic condition; and $R$ is defined as $R_{x}(\omega) R_{y}(\phi) R_{z}(\kappa)$, where $\omega$ denotes the rotation angle from positive $y$ axis to positive $\mathrm{z}$ axis, $\phi$ indicates the rotation angle from positive $z$ axis to positive $x$ axis, $\kappa$ is the rotation angle from positive $x$ axis to positive $y$ axis. The contents of the transformation matrix are indicated as follows:

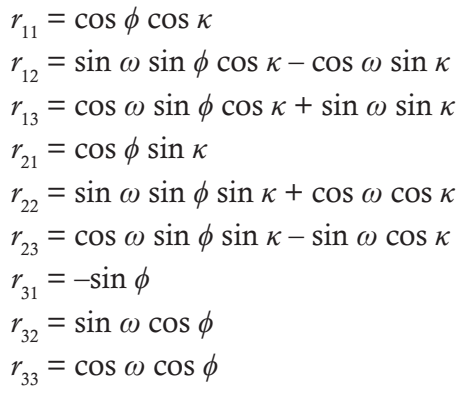

We reformulated Equation 1 after incorporating Equation 2 as Equation 3:

$E=\min \left(\sum_{i=1}^{n}\left\|\underline{p^{\prime}}-p_{i}^{\prime}\right\|^{2}\right)=\min \left(\sum_{i=1}^{n}\left\|R p_{i}+T-p_{i}{ }^{\prime}\right\|^{2}\right)$

Equation 3

The transformation matrix Tm was generated after solving Equation 3, and was further utilized to align the diastolic myocardium to the systolic coordinate system (Figure 1I). After registering, we established the strategy to maintain the relative position between the systolic and diastolic myocardium, thus comparing the displacement of each cardiac segment along the true short axis in the same coordinate system.

DIAMOND displacement vectors. We calculated the $3 \mathrm{D}$ mass centroid $\left(\mathrm{P}_{\mathrm{S}}\right.$ and $\left.\mathrm{P}_{\mathrm{D}}\right)$ coordinates $\overline{C_{k}}$ (where $k$ indicates the $x, y$, or $z$ coordinate, respectively) of each segment (I-VI) in the segmentation data set from systole to diastole (Figure $1 \mathrm{~J}$ ). We defined the mass centroid $\overline{C_{k}}$ in $3 \mathrm{D}$ space as follows:

$$
\overline{C_{k}}=\frac{1}{M_{i}} \sum_{j=1}^{m} C_{k j} \rho\left(x_{j}, y_{j}, z_{j}\right)
$$

\section{Equation 4}

where $C_{x}=x, C_{y}=y$, and $C_{z}=z ; M_{i}$ is the mass of each segment $(\mathrm{I} \leq i \leq \mathrm{VI}) ; m$ is interpreted as the number of voxels of each segment; and $\rho$ denotes the density function in which the segmented region $=1$, and the rest $=0$. The L2-norm of the sub-displacement vectors along the $x, y, z$ axes and the sum displacement vector were calculated during the cardiac cycle. The 3D cardiac movement of each ventricular segment was traced by quantifying the displacement of mass centroids during the cardiac cycle (Figure $1 \mathrm{~K}$ ). The segmental vectors (colored dots) of different portions are depicted in Figure 1L, indicating focal ventricular motion from end-systole to end-diastole. For normalization and expression of DIAMOND as percentage 
length change, the displacement value was divided by the end-systolic endocardial perimeter measured at the mid-ventricular long axis.

Transgenic zebrafish lines. The transgenic cardiac myosin light chain 2-monomeric Cherry Tg(cmlc2:mCherry) line, the transgenic cmlc2:mCherry combined with activated Notch Epstein-Barr virus terminal protein 1-green fluorescent protein reporter $T g(c m l c 2: m C h e r r y ; t p 1: g f p)$ line, and the transgenic fluorescent ubiquitylation-based cell cycle indicator $\mathrm{Tg}(\mathrm{cmlc2}$ :FUCCI) zebrafish line were used under the following conditions: (a) control, (b) doxorubicin treatment, and (c) doxorubicin combined with DAPT ( $\gamma$-secretase inhibitor) cotreatment. The transgenic $\mathrm{Tg}(\mathrm{cmlc2:mCherry)}$ zebrafish line was additionally used for NICD and NRG1 mRNA microinjections.

Chemical treatments. Doxorubicin (Sigma-Aldrich) was used at a concentration of $10 \mu \mathrm{M}$ in E3 fish water medium and for treatment at $3 \mathrm{dpf}$. After 24-hour treatment (4 dpf), doxorubicin medium was replaced with fresh E3 medium. For the experiment group doxorubicin + DAPT 3 dpf, the Notch $\gamma$-secretase inhibitor DAPT (Sigma-Aldrich) was used at a concentration of $10 \mu \mathrm{M}$ and cotreated with doxorubicin in E3 fish water medium at $3 \mathrm{dpf}$. After 24-hour treatment ( $4 \mathrm{dpf}$ ), doxorubicin + DAPT medium was replaced with $10 \mu \mathrm{M}$ DAPT-only medium. For the experiment groups doxorubicin + DAPT $4 \mathrm{dpf}$ and doxorubicin + DAPT $5 \mathrm{dpf}$, fish were placed in DAPT-only medium $(10 \mu \mathrm{M})$ at $4 \mathrm{dpf}$ and $5 \mathrm{dpf}$, respectively, after the removal of doxorubicin medium at $4 \mathrm{dpf}$.

Zebrafish cardiomyocyte isolation, treatment, and percent cell shortening. Adult zebrafish cardiomyocytes were isolated and cultured as previously described (21). Approximately 5000 cardiomyocytes were cultured per well in a 24-well plate. After 48 hours of incubation, zebrafish cardiomyocytes were transduced with NRG1 adenovirus (Vector Biolabs) with MOI of 100 for 6 hours, after which cells were placed in DMEM + FBS 10\% medium. Adenoviral transduction was verified by RT-PCR. Forty-eight hours after transduction, cardiomyocytes were treated with $10 \mu \mathrm{M}$ doxorubicin and $10 \mu \mathrm{M}$ DAPT, and imaged the following day using an inverted phase-contrast microscope (Olympus). Relaxed cardiomyocyte length $L_{1}$ and contracted cardiomyocyte length $L_{2}$ were manually measured with ImageJ as demonstrated in Supplemental Figure 5. Percent cell shortening was calculated as $\left(L_{1}-L_{2}\right) / \mathrm{L}_{1} \times 100$.

Confocal microscope imaging. Confocal microscopy with a lateral resolution of $\sim 0.57 \mu \mathrm{m}$ and a $z$-step of $\sim 2 \mu \mathrm{m}$ was used for focal analysis of Notch activation and cardiomyocyte proliferation. Images were taken at the UCLA Advanced Light Microscopy/Spectroscopy Core Laboratory using a Leica SP8-SMD confocal microscope.

$3 D$ quantification of Notch activation. The transgenic $T g(c m l c 2: m C h e r r y ; t p 1: g f p)$ zebrafish line was used to assess activated Notch expression (29). Endo- and epicardial Notch activation $(g f p)$ was delineated manually with Amira software, and the oversaturated signal originating from the atrioventricular valve was excluded from analyses. The 3D volume of Notch expression was calculated and normalized to the myocardial volume to account for size heterogeneity of zebrafish hearts. Segmental Notch expression was also analyzed.

Cardiomyocyte proliferation quantification. We adopted the FUCCI transgenic system to monitor proliferating cardiomyocytes in live zebrafish embryos, provided by Kenneth Poss (Duke University, Durham, North Carolina, USA) (30). The 2 transgenic lines used in the FUCCI system employ 2 fusion proteins, $T g(c m l c 2: m C h e r r y-z C d t)^{p d 57}$ and $T g$ (cmlc2-Venus-hGeminin $)^{p d 58}$, which were expressed cyclically in the $\mathrm{G}_{1}$ and $\mathrm{S} / \mathrm{G}_{2} / \mathrm{M}$ phase, respectively. Expression of the fusion proteins behind the cmlc2 promoter in stable transgenic lines, $\mathrm{Tg}(\mathrm{cmlc2}: \mathrm{mCherry}-z C d t 1)^{p d 57} ; \mathrm{Tg}(\mathrm{cmlc2} \text { :Venus-hGeminin })^{p d 58}$, identified cardiomyocytes at different cell cycle phases. Venus-hGeminin $\left(\right.$ green $\left.^{+}\right)$and mCherry-Zcdt1 (red $\left.{ }^{+}\right)$signals represent cells in S/ $\mathrm{G}_{2} / \mathrm{M}$ and $\mathrm{G}_{1}$ phases, respectively. Only red $\mathrm{d}^{-}$green $^{+}$nuclei were considered proliferating. $Z$-stacks of the heart were acquired using LSFM, and the maximum intensity projection of the $Z$-stacks was adopted to determine the percentage of proliferating cardiomyocytes. To evaluate the segmental cardiomyocyte proliferation, the $T g$ (cmlc2-Venus-hGeminin $)^{\text {pd58 }}$ line was crossed with the $T g$ (cmlc2:mCherry) line to delineate the myocardium. The analysis was performed by the same method adopted by DIAMOND, and the Z-stacks of the heart were acquired using a confocal microscope.

Quantification of trabeculation volume. To quantify changes in trabecular volume $\left(\mu \mathrm{m}^{3}\right)$, we reconstructed the zebrafish heart at end-systole after image synchronization (Amira software). Trabecular myocardium $\left(V_{T}\right)$ was manually labeled, and compact myocardium $\left(V_{c}\right)$ was determined by manually removing trabecular ridges. To account for the intrinsic variation of heart size between samples, trabecular myocardium volume ratio $\left[V_{T} /\left(V_{T}+V_{c}\right)\right]$ was employed for analyses.

Preparation and microinjection of NICD and NRG1 mRNA for Notch rescue. Rat NICD cDNA (Supplemental 
Table 2) and human NRG1 cDNA (a gift from William Talbot, Stanford University, Stanford, California, USA) (Supplemental Table 3) were amplified from donor plasmid and cloned into the plasmid pCS2+ at the BamHI/EcoRI sites. Clones with inserts of interest were selected by PCR screening. Two clones with human NRG1 cDNA insert were verified by transfecting the plasmids into HEK293 cells, and NRG1 protein expression was detected by Western blotting with anti-NRG1 antibody. mRNA was made using the mMessage SP6 Kit (Invitrogen) following the manufacturer's instructions. The in vitro transcribed mRNA was purified with Total RNA Isolation Kit (Bio-Rad) for in vivo rescue experiments. NICD mRNA at $10 \mathrm{pg} /$ $\mathrm{nl}$ and NRG1 mRNA at $5 \mathrm{pg} / \mathrm{nl}$ were injected at the 1-cell stage in zebrafish embryos to overexpress Notch target genes and to promote cardiac regeneration.

Code availability. The DIAMOND plugin module written in MATLAB (MathWorks) code is freely available at https://github.com/dingyichen1122/4-D-DIAMOND-Project.

Data availability. The datasets generated during and/or analyzed during the current study are available from the corresponding author.

Study approval. Zebrafish were maintained in accordance with the UCLA Institutional Animal Care and Use Committee (IACUC), and experiments were performed in compliance with protocols approved by the UCLA Office of Animal Research.

Statistics. All values are expressed as mean \pm SEM. For statistical comparisons between 2 experimental conditions, an unpaired 2-tailed $t$ test was used. For all other experimental conditions, comparisons of multiple means were performed by 1-way ANOVA, and statistical significance among multiple groups was determined using Tukey's method. GraphPad version 6 was used to perform statistical analyses. A $P$ value less than 0.05 was considered significant.

\section{Author contributions}

JC, YD, TKH, and RRSP designed the study; JC, YD, and RRSP developed the software; JC, YD, MC, JG, NJ, CN, ST, CC, SZ, CCC, JL, and RRSP acquired the data and validated the study; JC and RRSP analyzed the data; XX assisted with the chemo-induced injury animal model; JC, YD, and RRSP wrote the original manuscript draft; JC, YD, TKH, and RRSP reviewed and edited the manuscript; TKH and RRSP supervised the study; YD, TKH, and RRSP provided funding.

\section{Acknowledgments}

The present work was funded by NIH grants HL083015 (TKH), HL111437 (XX, TKH, RRSP), HL118650 (TKH), HL129727 (TKH), HL081753 (XX), and HL107304 (XX), American Heart Association grants 18CDA34110338 (YD) and 16SDG30910007 (RRSP), and the Cardiovascular Research Foundation of Southern California (RRSP).

Address correspondence to: Tzung K. Hsiai, Departments of Medicine (Cardiology) and Bioengineering, UCLA, 10833 Le Conte Ave., CHS 37-200G, Los Angeles, California 90095-1679, USA. Phone: 310.268.3839; Email: Thsiai@mednet.ucla.edu. Or to: René R. Sevag Packard, Department of Medicine (Cardiology), UCLA, 10833 Le Conte Ave., CHS 17-054A, Los Angeles, California 90095-1679. Phone: 310.825.4467; Email: RPackard@mednet.ucla.edu.

1. Ewer MS, Ewer SM. Cardiotoxicity of anticancer treatments. Nat Rev Cardiol. 2015;12(9):547-558.

2. Lenneman CG, Sawyer DB. Cardio-oncology: an update on cardiotoxicity of cancer-related treatment. Circ Res. 2016;118(6):1008-1020.

3. Burridge PW, et al. Human induced pluripotent stem cell-derived cardiomyocytes recapitulate the predilection of breast cancer patients to doxorubicin-induced cardiotoxicity. Nat Med. 2016;22(5):547-556.

4. Poss KD, Wilson LG, Keating MT. Heart regeneration in zebrafish. Science. 2002;298(5601):2188-2190.

5. Chamuleau SAJ, et al. Translational research in cardiovascular repair: a call for a paradigm shift. Circ Res. 2018;122(2):310-318.

6. MacRae CA, Peterson RT. Zebrafish as tools for drug discovery. Nat Rev Drug Discov. 2015;14(10):721-731.

7. Ding YH, et al. A modifier screen identifies DNAJB6 as a cardiomyopathy susceptibility gene. JCI Insight. 2016;1(14):e88797.

8. Karra R, Poss KD. Redirecting cardiac growth mechanisms for therapeutic regeneration. J Clin Invest. 2017;127(2):427-436.

9. Asnani A, et al. Highly potent visnagin derivatives inhibit Cyp1 and prevent doxorubicin cardiotoxicity. JCI Insight. 2018;3(1):96753.

10. Mickoleit M, et al. High-resolution reconstruction of the beating zebrafish heart. Nat Methods. 2014;11(9):919-922.

11. Fei P, et al. Cardiac light-sheet fluorescent microscopy for multi-scale and rapid imaging of architecture and function. Sci Rep. $2016 ; 6: 22489$ 
12. Lee J, et al. 4-Dimensional light-sheet microscopy to elucidate shear stress modulation of cardiac trabeculation. J Clin Invest. 2016;126(5):1679-1690.

13. Packard RRS, et al. Automated segmentation of light-sheet fluorescent imaging to characterize experimental doxorubicin-induced cardiac injury and repair. Sci Rep. 2017;7(1):8603.

14. Ding Y, et al. Integrating light-sheet imaging with virtual reality to recapitulate developmental cardiac mechanics. JCI Insight. 2017;2(22):e97180.

15. Lee J, et al. Spatial and temporal variations in hemodynamic forces initiate cardiac trabeculation. JCI Insight. 2018;3(13):e96672

16. Clarke SA, Richardson WJ, Holmes JW. Modifying the mechanics of healing infarcts: is better the enemy of good? J Mol Cell Cardiol. 2016;93:115-124.

17. Genet $\mathrm{M}$, et al. A novel method for quantifying smooth regional variations in myocardial contractility within an infarcted human left ventricle based on delay-enhanced magnetic resonance imaging. J Biomech Eng. 2015;137(8):081009.

18. Ogle BM, et al. Distilling complexity to advance cardiac tissue engineering. Sci Transl Med. 2016;8(342):342ps13.

19. Collier P, Phelan D, Klein A. A test in context: myocardial strain measured by speckle-tracking echocardiography. J Am Coll Cardiol. 2017;69(8):1043-1056.

20. Kruizinga P, Mastik F, Bosch JG, de Jong N, van der Steen AF, van Soest G. Measuring submicrometer displacement vectors using high-frame-rate ultrasound imaging. IEEE Trans Ultrason Ferroelectr Freq Control. 2015;62(10):1733-1744.

21. Sander V, Suñe G, Jopling C, Morera C, Izpisua Belmonte JC. Isolation and in vitro culture of primary cardiomyocytes from adult zebrafish hearts. Nat Protoc. 2013;8(4):800-809.

22. Cain P, Baglin T, Khoury V, Case C, Marwick TH. Automated regional myocardial displacement for facilitating the interpretation of dobutamine echocardiography. Am J Cardiol. 2002;89(12):1347-1353.

23. Ding Y, et al. Multiscale light-sheet for rapid imaging of cardiopulmonary system. JCI Insight. 2018;3(16):e121396.

24. Power RM, Huisken J. A guide to light-sheet fluorescence microscopy for multiscale imaging. Nat Methods. 2017;14(4):360-373.

25. Amzulescu MS, et al. Improvements of myocardial deformation assessment by three-dimensional speckle-tracking versus two-dimensional speckle-tracking revealed by cardiac magnetic resonance tagging. J Am Soc Echocardiogr. 2018;31(9):1021-1033.e1

26. Grego-Bessa J, et al. Notch signaling is essential for ventricular chamber development. Dev Cell. 2007;12(3):415-429.

27. Kasahara A, Cipolat S, Chen Y, Dorn GW, Scorrano L. Mitochondrial fusion directs cardiomyocyte differentiation via calcineurin and Notch signaling. Science. 2013;342(6159):734-737.

28. Zhao L, et al. Notch signaling regulates cardiomyocyte proliferation during zebrafish heart regeneration. Proc Natl Acad Sci USA 2014;111(4):1403-1408.

29. Parsons MJ, et al. Notch-responsive cells initiate the secondary transition in larval zebrafish pancreas. Mech Dev. 2009;126(10):898-912.

30. Choi WY, et al. In vivo monitoring of cardiomyocyte proliferation to identify chemical modifiers of heart regeneration. Develop ment. 2013;140(3):660-666.

31. Fukazawa R, et al. Neuregulin-1 protects ventricular myocytes from anthracycline-induced apoptosis via erbB4-dependent activation of PI3-kinase/Akt. J Mol Cell Cardiol. 2003;35(12):1473-1479.

32. Natarajan N, et al. Complement receptor C5aR1 plays an evolutionarily conserved role in successful cardiac regeneration. Circulation. 2018;137(20):2152-2165. 